\section{PRESIDENTIAL ROUND UP}

Bertie Napier is the new President of the British Academy of Cosmetic Dentistry. He replaces outgoing President Anoop Maini.

Jane Dalgarno is the new President of the British Association of Dental Nurses. She replaces outgoing President Fiona Ellwood.

Dr Robin Mills is the new President of the British Society of Paediatric Dentistry. He replaces outgoing President Dr Janice Fearne.

\section{BOOK REVIEW

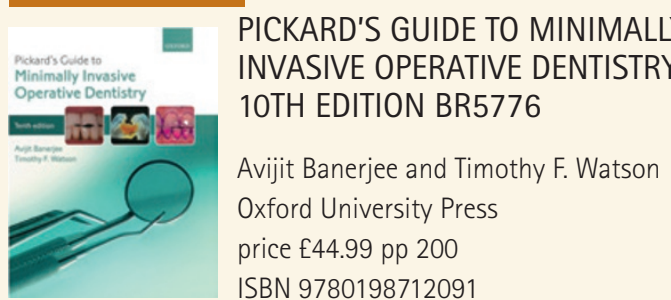

'Pickard's Guide' has long been held in high regard, as one of the mainstays for simple operative dentistry and the latest edition certainly doesn't disappoint.

The book provides an excellent overview of the full range of topics related to this area of dentistry and is cleverly structured into well-defined sections. Each chapter begins with a short introduction including clear objectives, allowing the reader to understand what information will be covered. Alongside this, each chapter is dotted with 'self-test' questions; the answers to which are included at the end of each section, encouraging the reader to develop and test their knowledge as they go.

The book starts with an introduction to dental hard tissue pathologies, covering caries, tooth wear, developmental defects and trauma, and providing clear descriptions and definitions of key terms. Aside from the technical aspects of operative dentistry, this useful book also includes chapters providing information on disease prevention, infection control and aesthetics.

Although the book covers a wide range of information, it is easy to read with numerous useful diagrams and high quality pictures on each page to help illustrate the information. Towards the end of the book there is a chapter, which includes a 33-page section describing each operative technique, with short texts and pictures that result in an informative step-by-step guide.

The tenth edition is updated to include more modern restorative techniques, as well as an emphasis on the differing important roles of the entire oral healthcare team. They have also added technology in the form of $Q R$ codes, enabling the reader to easily scan and access the supporting material through digital media.

Overall this book addresses the core competencies relevant to undergraduates in training and would be beneficial to such readers. Additionally it would be beneficial to oral healthcare professionals looking for an ideal overview of the theory and practical aspects of conservative dentistry.

NAOMI PRADO

\title{
RECOGNISING THE EARLY STAGES OF DEMENTIA THROUGH ORAL HEALTH
}

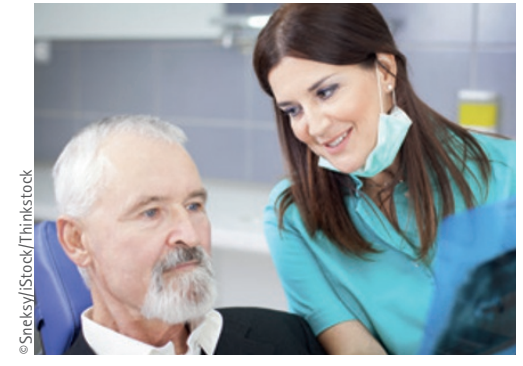

People in the early stages of dementia may be able to be identified by dentists through the state of their oral health, according to the British Dental Health Foundation (BDHF).

The oral health charity says that many people with dementia are often unable to properly maintain their oral health themselves as their health declines and this could be a key signal for dentists and doctors of the early stages of the disease.

The BDHF believes that offering people with dementia plans for effective healthcare could ensure their oral health, and overall health, is not put at further risk.

Chief Executive of the BDHF, Dr Nigel Carter OBE, discussed how this could lead to better oral healthcare for people with dementia: 'Dementia patients in early stages of the disease may have trouble communicating the problems they are having with their oral health.

'These patients need to rely on their dental professionals to recognise behaviour which is out of the ordinary and which may indicate mental health problems in order to get quick and effective support.

'As dementia is progressive, recognising it early means that an effective care-plan can be put into place before it leads to further health problems, including painful and extensive dental health issues.

'Through proper maintenance of oral health in people with dementia there are many other benefits in terms of self-esteem, dignity and nutrition. What we must remember is that the impact of proper and improper oral health is no different for those who have and don't have dementia, and should be treated as accordingly.'

The Alzheimer's Society estimate that there are currently more than
850,000 people with dementia in the UK and this will be over one million by 2025 . Studies have shown that people with dementia have poorer oral health than those without the disease due to impairment of cognitive skills and a reliance on care providers.

George McNamara, Head of Policy and Public Affairs at Alzheimer's Society said: 'We know how important getting a timely diagnosis of dementia is, enabling access to treatments and support, and acting as a catalyst for discussions about how to live with dementia.

'If dental practitioners can help to spot the early signs of dementia through monitoring any deterioration in oral health, and help people to be diagnosed more quickly, that can only be a good thing.

'We look forward to working with the dental profession to help them spot the warning signs of dementia and then, where appropriate, refer patients to their GP for further investigation.

'Dentists have an important role to play in the identification of people showing symptoms of dementia. There needs to be greater integration between dentists and GPs to ensure information, advice and appropriate signposting takes place so that people with dementia can receive a timely diagnosis.

'Alzheimer's Society recognises how important an oral healthcare programme is for people with dementia. This programme should be established as early as possible so that people can independently maintain their oral health for as long as possible.

'Once the condition progresses their carer will need to support them in keeping their teeth healthy and a dentist should enable this to happen. Furthermore people with dementia in advanced stages may not be able to communicate that they are experiencing pain in their teeth so dentists should be trained to recognise other signs which may indicate an underlying problem. With appropriate training dentists will be able to help people with dementia maintain a good quality of oral health and reduce discomfort.' 